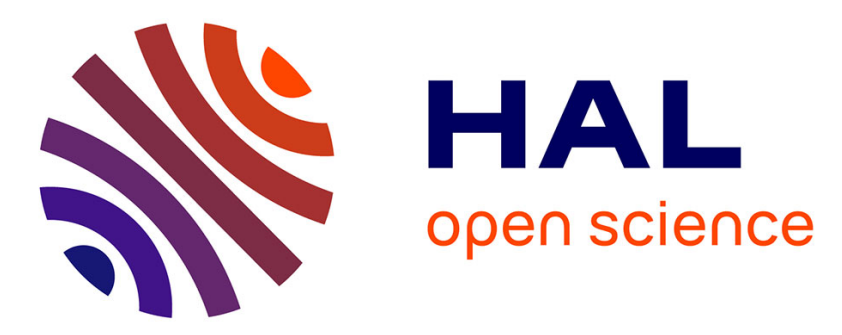

\title{
Sap flow measurements by thermal dissipation method using cyclic heating: a processing method accounting for the non-stationary regime
}

Nourtier, Andre Chanzy, Granier, Huc

\section{- To cite this version:}

Nourtier, Andre Chanzy, Granier, Huc. Sap flow measurements by thermal dissipation method using cyclic heating: a processing method accounting for the non-stationary regime. Annals of Forest Science, 2011, 68 (7), pp.1255-1264. 10.1007/s13595-011-0065-3 . hal-00930799

\section{HAL Id: hal-00930799 \\ https://hal.science/hal-00930799}

Submitted on 1 Jan 2011

HAL is a multi-disciplinary open access archive for the deposit and dissemination of scientific research documents, whether they are published or not. The documents may come from teaching and research institutions in France or abroad, or from public or private research centers.
L'archive ouverte pluridisciplinaire HAL, est destinée au dépôt et à la diffusion de documents scientifiques de niveau recherche, publiés ou non, émanant des établissements d'enseignement et de recherche français ou étrangers, des laboratoires publics ou privés.

$$
\text { Copyright }
$$




\title{
Sap flow measurements by thermal dissipation method using cyclic heating: a processing method accounting for the non-stationary regime
}

\author{
Marie Nourtier • André Chanzy • André Granier • \\ Roland Huc
}

Received: 10 February 2011 /Accepted: 17 March 2011 /Published online: 6 May 2011

(C) INRA and Springer Science+Business Media B.V. 2011

\begin{abstract}
- Context The thermal dissipation method to measure sap flow in tree stems can be used with cyclic heating to reduce electricity consumption and/or to account for natural temperature gradients. Nevertheless, errors in sap flow estimation can be introduced because the thermal equilibrium has not been reached at the measurement time.

- Aim We propose a method to assess this error and to estimate sap flow density. It is based on Granier's (Ann For Sci, 42:193-200, 1985) non-species-specific calibration.

- Methods This work was performed on silver fir trees (Abies alba Mill.) with low sap flux densities (maximum of $0.68 \mathrm{Ldm}^{-2} \mathrm{~h}^{-1}$ ). To estimate the error, we developed a calibration procedure using the experimental set-up in trees. This approach is based on a pair of sensors having similar temporal patterns in sap flux density, one being under cyclic heating while the other is continuously heated.

- Results Applying Granier's calibration without correction led to large errors (relative error reached 200\%). After correction, the error was greatly diminished; it was lower
\end{abstract}

\section{Handling Editor: Gilbert Aussenac}

M. Nourtier · A. Chanzy $(\triangle)$

INRA-UMR1114, INRA/UAPV, Environnement Méditerranéen

et Modélisation des AgroHydrosystèmes,

Site Agroparc,

84914 Avignon, France

e-mail: achanzy@avignon.inra.fr

A. Granier

INRA-UMR1137, Écologie et Écophysiologie forestière,

54280 Champenoux, France

R. Huc

INRA-UR629, Écologie des Forêts Méditerranéennes,

Site Agroparc,

84914 Avignon, France than $0.042 \mathrm{Ldm}^{-2} \mathrm{~h}^{-1}$ when using short heating cycles $(0.5 \mathrm{~h})$.

- Conclusion The correction was applicable to all silver fir trees monitored. However, this method can be easily repeated to investigate the validity domain of the correction.

Keywords Sap flow - Thermal dissipation method - Cyclic heating . Error correction $\cdot$ Silver fir

\section{Introduction}

Sap flow measurement is the only method to assess water fluxes in the soil-vegetation-atmosphere continuum at the tree scale or over complex terrain where the soil water balance or micrometeorological methods cannot be implemented. There are several techniques for measuring sap flow (Köstner et al. 1998), including heat pulse velocity, tissue heat balance (Čermák-type) and radial flowmetry (Granier type). Granier's method (Granier 1985, 1987) is one of the most commonly used for trees because of its simplicity, low cost and reliability (Andrade et al. 1998; Braun and Schmid 1999; Do and Rocheteau 2002a; Lu et al. 2004). This technique uses a thermal dissipation probe radially inserted into the stem. The equation for calculating sap flux density is applicable to every tree species when the sensor geometry and electrical power remain identical and when the entire length of the probe is surrounded by the hydroactive xylem, creating a high thermal conductivity between the probe and the wood (Granier 1987; Lu et al. 1996; $\mathrm{Lu}$ et al. 2004). The main drawback of the Granier method is its relatively high electricity consumption, which may be a critical issue in the experimental design because several repetitions are needed to scale up the measurements made at the tree level to an entire forest stand. 
Granier (in Köstner et al. 1998) first proposed cyclic heating to diminish power consumption. Do and Rocheteau $(2002 a, b)$ further developed the cyclic heating method to reduce both power consumption and the effect of ambient thermal gradients. However, cyclic heating limits temporal resolution. Therefore, using a cyclic heating method implies making a trade-off between spatial and temporal sampling. This method also requires recalibration because the thermal stationary regime is not always reached at the end of a heating or cooling cycle. Do and Rocheteau (2002a, b) established a non-species-specific calibration (D\&R calibration) fitted to non-stationary regimes using short cycles. The D\&R calibration was built with measurements on artificial columns filled with sawdust and was confirmed on tropical trees by Isarangkool Na Ayutthaya et al. (2010). However, they found an overestimation of $30 \%$ under low flow conditions and proposed an updated calibration relationship. The time needed to reach the stationary regime is determined by the heat exchanges between probes, wood and xylem sap. Therefore, this time may depend on the tree species, the sap flow rate and the trunk diameter. It is possible, then, that $\mathrm{D} \& \mathrm{R}$ calibration cannot be generalised to all tree species and dimensions.

The aim of this study was to assess whether cyclic heating can be applied to measure sap flow with the Granier method on silver fir trees with low sap flow rates. Unlike the D\&R calibration approach, which is based on a specific calibration for cyclic measurements, we used the calibration relationship proposed by Granier (1985). Use of this relationship is strongly supported by its successful application with numerous tree species. A primary objective of the present study was to analyse how a non-stationary regime impacts temperature measurements and the resulting sap flow calculations in relation to the timing of the heating cycle. A generic correction method, based on in situ measurements, was then developed and evaluated. This correction method involves building parameters from the previous error assessment to extrapolate the non-stationary regime to the stationary regime. Finally, a comparison was made with the D\&R calibration method.

\section{Materials and methods}

\subsection{Granier's method}

Sap flow is measured with a thermal dissipation sap flowmeter composed of two probes. These probes are inserted radially into the xylem, just beneath the tree bark. One of the two probes is heated with a constant energy input, while the other is not heated and remains at the same temperature as that of the wood. Sap flux density is a function of the temperature difference $(\Delta T)$ between the two probes. Under no-flow conditions, the temperature of the heated probe stabilises at a maximum value, $\Delta T_{0}$, when heat dissipation by conduction through the wood equals the heat input. An accurate measurement of $\Delta T_{0}$ is necessary for the calculation of sap flux density. Under no-zero flow conditions, heat convection increases and $\Delta T$ decreases. Granier (1985) calibrated the following relationship to estimate sap flux density $\left(J_{t}\right.$ in litres per square decimetres per hour) at time $t$ :

$J_{t}=\alpha \cdot\left(\frac{\Delta T_{0}-\Delta T_{t}}{\Delta T_{t}}\right)^{\beta}=\alpha K^{\beta}$

where $\alpha=4.2841 \mathrm{Ldm}^{-2} \mathrm{~h}^{-1}, \beta=1.231, \Delta T_{0}$ is the maximum temperature difference between both probes under zero flow conditions and $\Delta T_{t}$ is the temperature difference between probes at time $t$.

\subsection{Experimental design}

This study was conducted on silver fir (Abies alba Mill.) trees at Mont Ventoux (southern France, $44^{\circ} 10^{\prime} 28^{\prime \prime}$ N, $5^{\circ} 16^{\prime}$ $44^{\prime \prime}$ E), an area with both mountain and Mediterranean climate conditions. Four trees, separated by a few metres, were chosen in a plot at $1,360 \mathrm{~m}$ in elevation. On average, the trees were $18 \mathrm{~m}$ in height and $38 \mathrm{~cm}$ in diameter at breast height. On one tree, two sensors were inserted at breast height between 0 - and $2-\mathrm{cm}$ depth at the north and west azimuths. Care was taken to place the sensors far enough apart to avoid interference between the probes. On the three other trees, one sensor was inserted at the north azimuth at breast height (Table 1). Additional information on the studied trees is given in Table 1.

In another plot at $1,020 \mathrm{~m}$ in elevation, one tree was instrumented with three sensors at the north, east and west azimuths, and two other sensors were installed on the north sides of two other tree stems. These trees were included in the experimental design to test the validity of the correction.

2.3 Characterisation of the impact of non-stationary regime on $\Delta T$ measurement and sap flow calculation

The aim of the experiment was to characterise how far a sensor's thermal regime from the stationary condition is at the end of the heating cycle and what the consequences are for sap flow calculation when Granier's calibration is used. To achieve these aims, we designed a specific measurement configuration based on the use of two sensors with similar temporal behaviours, one being heated continuously and the other with cyclic heating.

Because it can take a long time to reach a stationary regime, waiting for this moment compromises the time 
Table 1 Characteristics of the investigated trees and of the set-up of the sensors, and mean differences of the sap flux density variations between all sensors

\begin{tabular}{|c|c|c|c|c|c|}
\hline & Sensor 1 & Sensor 2 & Sensor 3 & Sensor 4 & sensor 5 \\
\hline Sensor 1 & & 0.065 & 0.056 & 0.030 & 0.017 \\
\hline Sensor 2 & & & 0.009 & 0.053 & 0.050 \\
\hline Sensor 3 & & & & 0.042 & 0.039 \\
\hline Sensor 4 & & & & & 0.017 \\
\hline Azimuth on the stem & $\mathrm{N}$ & $\mathrm{W}$ & $\mathrm{N}$ & $\mathrm{N}$ & $\mathrm{N}$ \\
\hline Stem diameter at breast height $(\mathrm{cm})$ & 28.5 & 44.1 & 44.1 & 42 & 40.1 \\
\hline Mean sap flux density $\left(\mathrm{L} \mathrm{dm}^{-2} \mathrm{~h}^{-1}\right)$ & 0.30 & 0.22 & 0.24 & 0.32 & 0.24 \\
\hline
\end{tabular}

Sensors 1 and 4 form pair 1 . Sensors 2 and 3 are those on the same trees and form pair 2 . Sensors 3 and 4 are the continuously heated sensors

resolution of measurements from cyclic heating. Therefore, we wanted to estimate the error that arises when performing $\Delta T$ measurements before the stationary condition is reached. This error is defined as the difference between $\Delta T_{t \text {-cyclic }}$ at time $t$, corresponding to the end of a heating cycle, and a reference $\Delta T_{\text {ref, }}$ which is the value of $\Delta T$ that would be obtained if the stationary regime was reached. The difficulty resides in the estimation of this last term, which must be determined with the same sensor to avoid sap flow variability between sensors installed either on the same tree or on different trees. In this study, $\Delta T_{\text {ref }}$ was established by extending the heating period until $t_{\mathrm{m}}$, the time required to reach the stationary regime. This time depends on the heat exchanges between probes, wood and xylem sap. This length of time increases when the sap flow is low. This is shown in Fig. 1, in which the $\Delta T$ variations obtained at night and near midday are displayed. The figure clearly shows that after $1 \mathrm{~h}$ of heating, the thermal stationary regime had not been reached and that the $\Delta T$ stabilised more quickly when fluxes were high. To be confident that the stationary conditions were reached, the heating period $\left(t_{\mathrm{m}}\right)$ during the night was set to $6 \mathrm{~h}$ (after a 3-h non-heating period), while it was set to $t_{\mathrm{m}}=3 \mathrm{~h}$ (after a 2-h non-heating period) during the day. We found in the night measurements that when the sap flow was close to zero, $3 \mathrm{~h}$ of heating was sufficient to reach the stationary regime, thus confirming that the selected $t_{\mathrm{m}}$ was appropriate.

The difference in temperature at $t_{\mathrm{m}}\left(\Delta T_{t_{m}}\right)$ is not a direct estimator of $\Delta T_{\text {ref }}$ because the $\Delta T$ may vary between $t$ and $t_{\mathrm{m}}$ due to the natural daily sap flow fluctuations. To estimate such variations, we used the continuously heated sensor. On this sensor, the $\Delta T$ values were selected at the same times, $t$ and $t_{\mathrm{m}}$ (Fig. 2).

The errors were calculated separately for both terms of Eq. 1: $\Delta T_{0}$ and $\Delta T_{t}$. The error is defined as the difference between $\Delta T_{\text {ref }}$ and the value of $\Delta T_{t \text {-cyclic }}$, measured at the end of the heating cycle. The value for the $\Delta T_{\text {ref }}$ is estimated by $\Delta T_{t_{m}}$ (measured by the sensor under cyclic heating) which is corrected by the natural difference resulting from the transpiration course derived from the continuously heated sensor (Fig. 2). The error $\left(E_{\Delta T}\right)$ was estimated for days and nights separately:

$E_{\Delta T}=\Delta T_{\text {ref }}-\Delta T_{t-\text { cyclic }}$

with

$\Delta T_{\text {ref }}=\Delta T_{t_{m}-\text { cyclic }}-\left(\Delta T_{t_{m}}-\Delta T_{t}\right)_{\text {continuous }}$

giving

$E_{\Delta T}=\left(\Delta T_{t_{m}}-\Delta T_{t}\right)_{\text {cyclic }}-\left(\Delta T_{t_{m}}-\Delta T_{t}\right)_{\text {continuous }}$

Sap flux density derived from cyclic heating is then corrected as follows:

$J_{t_{-} \text {corr }}=\alpha \cdot\left(\frac{\left(\Delta T_{0}+E_{\Delta \mathrm{T} 0}\right)-\left(\Delta T_{t}+E_{\Delta \mathrm{T} t}\right)}{\Delta T_{t}+E_{\Delta T t}}\right)^{\beta}$

where $E_{\Delta T_{0}}$ is the error in probe temperature difference during the night and $E_{\Delta T_{t}}$ during the day.

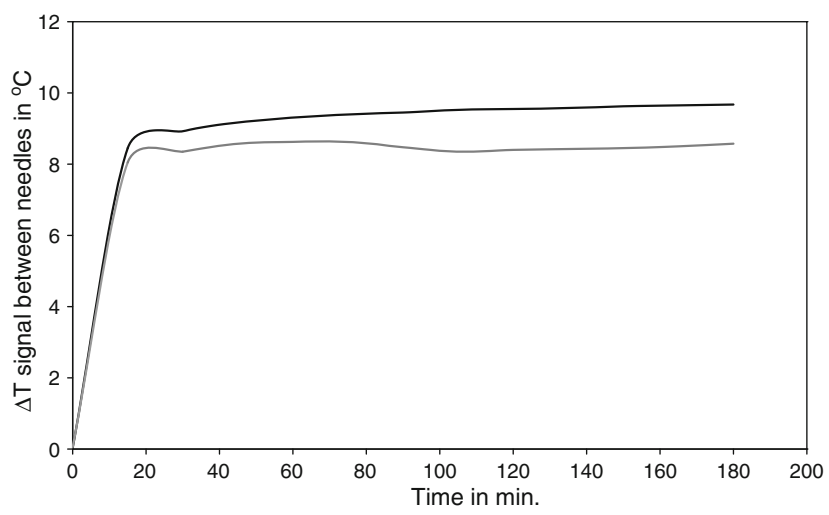

Fig. 1 Kinetics of sensor temperature difference during the nonstationary period (1) under zero flow conditions (black line) and (2) under medium, midday flow conditions, $J=0.7 \mathrm{~L} \mathrm{~m}^{-2} \mathrm{~h}^{-1}$ (grey line) 


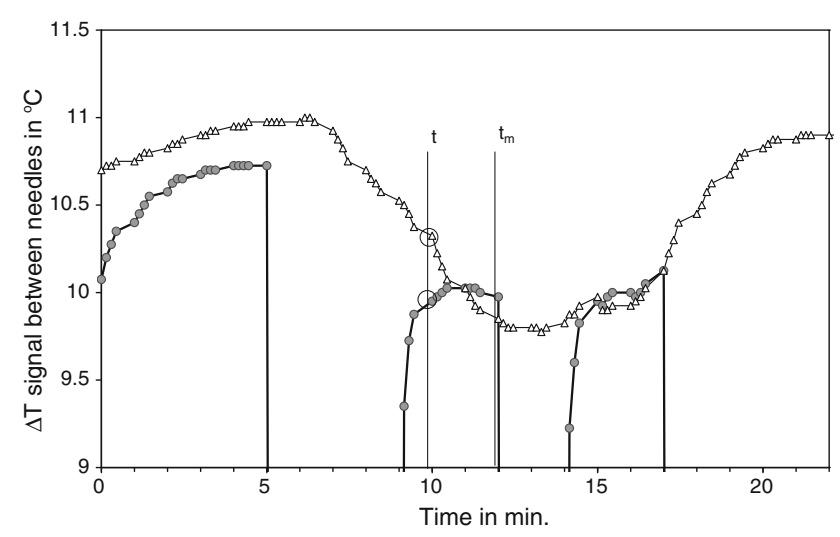

Fig. 2 Illustration of the error calculation method over a day. Grey points and line represent cyclic heating and black triangles and line represent continuous heating. Frequency of measurements is at $15-\mathrm{min}$ intervals. In this example, measurement at time $t$ is performed $1 \mathrm{~h}$ after turning on the heat

The effectiveness of this correction on sap flux density is determined by a comparison of the errors made before and after correction. Errors are calculated as follows:

$E_{J}=\left(J_{m}-J_{t}\right)_{\text {cyclic }}-\left(J_{m}-J_{t}\right)_{\text {continuous }}$

$E_{J_{-} \text {corr }}=\left(J_{m}-J_{t_{-} \text {corr }}\right)_{\text {cyclic }}-\left(J_{m}-J_{t}\right)_{\text {continuous }}$

where $E_{J}$ is the error in the sap flux density calculation before the correction, expressed in litres per square decimetre per hour, $E_{J_{-} \text {corr }}$ is the error in the sap flux density calculation after correction, expressed in litres per square decimetre per hour, $J_{m}$ is the sap flux density at the stationary regime and $J_{t}$ is the sap flux density calculated from the cyclic or continuous heating methods at the end of a given cycle, both in litres per square decimetre per hour.

We performed the measurements over 15 days. Three heating cycles were monitored per day: one during the night and the other two during the day. Different combinations of night and day cycles were tested. During each heating period, the values of $\Delta T_{t \text {-cyclic }}$ were retained at times $t=1,3$, and $5 \mathrm{~h}$ after heating began during the night and $t=0.5,1,1.5$, and $2 \mathrm{~h}$ after heating began during the day.

As with the D\&R calibration, a direct relationship between $K$, computed with the uncorrected cyclic $\Delta T$, and the reference sap flux density, $J_{t}$, calculated from continuously heated sensors, would have been possible.

\subsection{Comparison of sap flow dynamics between sensors}

The method for assessing error and developing a correction technique assumes the use of a pair of sensors presenting similar variations in sap flux density between $t$ and $t_{m}$. This similarity was assessed by monitoring the sap flow dynamics during a 3-month period with all of the sensors installed on the five trees of the plot at $1,360 \mathrm{~m}$. The sap flow dynamic of each sensor was characterised by the variation in sap flux density (using a $1-\mathrm{h}$ heating and $1 \mathrm{~h}$ cooling protocol without correction) between 10:00 a. m. and 12:00 p.m. and between 12:00 p.m. and 4:00 p.m. The differences in these variations between the two sensors of each pair were calculated for each day and at each period. The results were then averaged over a 3month period for each pair. The time intervals of 2 and $4 \mathrm{~h}$ were chosen to be representative of or larger than the time lag between $t$ and $t_{m}$. Moreover, the selected periods of measurement corresponded to periods of high sap flux variation during the day.

\subsection{Validation of the applicability of the corrections} on other trees

The rationale of the correction method is to estimate the error in $\Delta T$ when conditions are non-stationary at the end of the heating period. To further assess the validity of this approach, we evaluated whether the increase in the $\Delta T$ during the heating process is mainly driven by sap flux, implying that the thermal properties of the wood, the quality of the probe contact and local site influences are second-order factors. We made this evaluation on ten sensors installed on the seven different silver fir trees located at the two Mont Ventoux sites presenting different climatic conditions (1,360 and $1,020 \mathrm{~m}$ in elevation) and with similar diameters (ranging from 30 to $45 \mathrm{~cm}$ ). The measurements were carried out for more than 6 months in 2009. From these measurements, we computed the difference in $\Delta T$ after $15 \mathrm{~min}$ vs. $1 \mathrm{~h}$ of heating. This difference can be considered as an indicator of the heat exchange at the sensor level and the time required to reach stationary conditions. The results were related to the sap flux density calculated without making the proposed correction of $\Delta T$.

\subsection{Data analysis}

Analysis of the data was made by computing the following statistical quantities: the mean absolute error, to estimate the accuracy of the measurements; the standard deviation, to characterise the error distribution and the bias, to assess systematic errors. The mean relative error (absolute error divided by sap flux density) is also displayed to represent the weight of the error in comparison to the measurements. However, under low flux conditions, the relative error may be particularly strong. As the fluxes were often very low, interpretations of the mean relative error should be made with caution. 


\section{Results}

\subsection{Comparison of sap flow dynamics}

The mean differences in sap flux density variations were calculated and are displayed in Table 1 . The best results were achieved when the sensors were on the same tree. In this case, the difference in sap flux density variations was very low and corresponded to a low proportion of mean sap flux density (4\%). For the other pairs, this proportion was higher and varied between $7 \%$ and $25 \%$ of the mean sap flux density of the pair. These results highlight the difficulties in determining a priori which trees had similar sap flow dynamics. Using a pair of sensors installed on the same tree is therefore recommended. In the following sections, analyses were done considering two pairs of sensors: one installed on trees with similar sap flux density dynamics (pair 1 in Table 1) and the other installed on the same tree (pair 2 in Table 1).

\subsection{Estimation of sap flux density error}

During the night, the error made in temperature difference between sensors, $E_{\Delta T_{0}}$, appeared to depend only on the duration of heating (Table 2). This can be explained by a very low and stable night sap flow. The longer the heating period and the closer the conditions of measurement are to the stationary regime, the lower the error is. For instance, with a heating period of $5 \mathrm{~h}$, we obtained a low average absolute and constant error, $E_{\triangle T 0}=0.03^{\circ} \mathrm{C}$. With a 1h heating, the mean $E_{\Delta T_{0}}$ was much higher but was also relatively stable, with a standard deviation representing $17 \%$ of the mean error (Table 2). We did not find obvious factors explaining $E_{\Delta T_{0}}$ variability, and it was therefore considered to be constant.

During the day, $E_{\Delta T_{t}}$ depended on the sap flow rate $\left(J_{t}\right)$. For a given combination of night and day heating duration, the relationship between the error before correction, $E_{\Delta T_{t}}$, and the sap flux density is linear. Because $J_{t}$ is calculated from $\Delta T$ measured during the night and during the day, the coefficients of the relationship are different according to the heating durations of both night and day. The linear relationships obtained with the two pairs of sensors were

Table 2 Mean absolute error in $\Delta T_{0}$ during the night for increasing heating durations, in degrees Celsius

\begin{tabular}{lcr}
\hline Night heating duration & \multicolumn{1}{l}{ Pair 1 } & \multicolumn{1}{l}{ Pair 2 } \\
\hline $1 \mathrm{~h}$ & $0.42(0.12)$ & $0.51(0.085)$ \\
$3 \mathrm{~h}$ & $0.088(0.048)$ & $0.12(0.053)$ \\
$5 \mathrm{~h}$ & $0.029(0.024)$ & $0.025(0.017)$ \\
\hline
\end{tabular}

Standard deviations are in parentheses found to be identical, and a single set of coefficients is shown in Fig. 3 and Table 3. This demonstrates that the lack of accuracy in the choice of sensors to form pair 1 had little consequence on the $E_{\Delta T_{t}}=f\left(J_{T}\right)$ relationship. For 1.5- and 2-h heating cycles during the day, the error can be considered to be constant.

When considering the value of the $\Delta T$ between sensors before reaching the stationary regime, the mean error, $E_{J}$, in the sap flux density calculation was important: it could lead to an average error of $0.17 \mathrm{Ldm}^{-2} \mathrm{~h}^{-1}$ for pair 1 and $0.16 \mathrm{~L}$ $\mathrm{dm}^{-2} \mathrm{~h}^{-1}$ for pair 2 (Table 4), which are high in comparison to the mean sap flux density measured on continuously heated sensors during the experiment: $0.22 \mathrm{Ldm}^{-2} \mathrm{~h}^{-1}$ for sensor 4 of pair 1 and $0.25 \mathrm{Ldm}^{-2} \mathrm{~h}^{-1}$ for sensor 3 of pair 2. Depending on the combination of day and night cycles and on sap flux conditions, a lack of correction would lead to an overestimation or an underestimation (Table 4). The error was higher for the short cycles of the day $(0.5$ and $1 \mathrm{~h}$ of heating) except when the duration of night heating was $1 \mathrm{~h}$. In this case, the errors in night and day measurements were balanced, and the compensation led to a small error in the fluxes (Table 4). The best results were obtained with a heating period equal to or longer than $1.5 \mathrm{~h}$ during the day and $3 \mathrm{~h}$ during the night. Because there was a poorer assessment of the natural temporal $\Delta T$ variation for pair 1 compared to the estimation for pair 2, there was an overestimation of the error for pair 1 .

\subsection{Correction of the sap flux density}

In Fig. 3, the linear relationship between $E_{\Delta T_{t}}$ and $J_{t}$ suggests that $E_{\Delta T_{t}}$ can be estimated as follows, and as shown in Table 2, we can assume that $E_{\Delta T 0}$ is constant:

$E_{\Delta T t} \cong a . J_{t}+b$

$E_{\Delta T 0} \cong c$

where $a$ and $b$ are derived from the linear regression equation, $J_{t}$ is the sap flux density calculated before correction and $c$ is the mean constant error calculated for the night-time.

The results of the evaluation of the error after correction are only presented for pair 2, the pair for which the reference was more accurately estimated. The correction was done using coefficients from the linear regression fitted to this pair.

With the coefficients from the regression, the mean error in the sap flux density calculations decreased after the correction for each tested cycle (Table 5). There was a clear overall improvement: the maximum mean error was reduced almost by a factor of 4 . For cycles with the longest periods of heating ( 3 or $5 \mathrm{~h}$ of night heating and $2 \mathrm{~h}$ of day 
Fig. 3 Relationships between day error, $E_{\Delta T_{t}}$, and sap flux density, $J_{t}$, before correction for each day heating cycle (corresponding to indications of time on the graph) and for $3 \mathrm{~h}$ of heating during the night. Results are presented for the two pairs (pair 1, black unfilled circles; pair 2, filled circles). Black line shows the linear regression for both pairs 1 and 2. Coefficients of regression are in Table 3
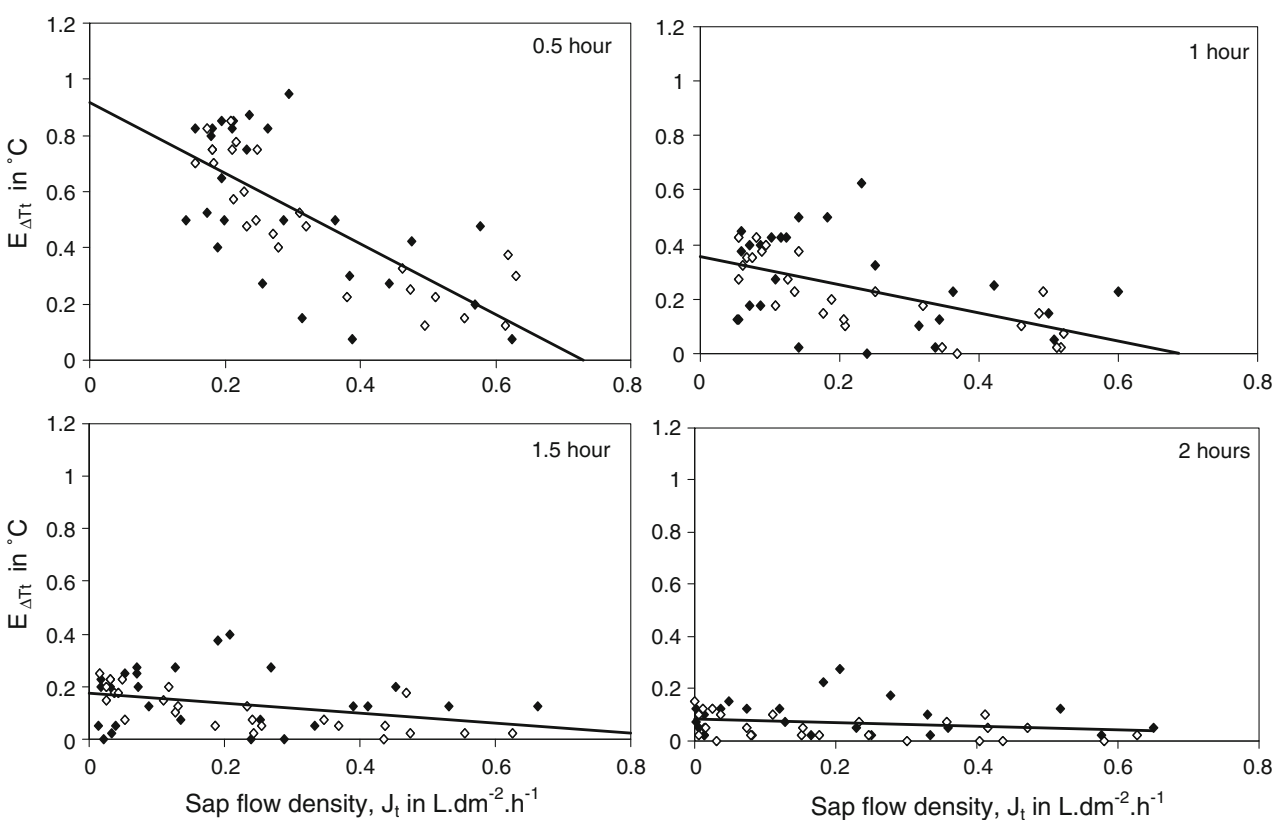

heating), little improvement was obtained, with the error only reduced by $10 \%$, but the errors were already much lower before the correction than those obtained for shorter heating cycles. For these cycles, the improvement was greater, leading to a remaining error close to that of the longest heating cycle (Tables 4 and 5). The remaining mean absolute error was always lower than $0.042 \mathrm{Ldm}^{-2} \mathrm{~h}^{-1}$ (Table 5), which should be compared to the mean sap flux density over the experiment, at $0.23 \mathrm{Ldm}^{-2} \mathrm{~h}^{-1}$, and to the maximum sap flux density, at $0.68 \mathrm{Ldm}^{-2} \mathrm{~h}^{-1}$. After the correction, the mean relative error was lower than $30 \%$ in most cases (Table 5) while it could represent more than $100 \%$ of the reference sap flux density before the correction (Table 4). The accuracy is comparable to that obtained by Do and Rocheteau (2002b) and Isarangkool Na Ayutthaya et al. (2010). In the latter, a relative error of $41 \%$ was found when sap fluxes were lower than $0.5 \mathrm{Ldm}^{-2} \mathrm{~h}^{-1}$.

In most cases, the value of the bias was close to the mean absolute error (Table 5). This means that, after correction, there was a systematic overestimation of the sap flux density. The bias decreased with an increase of the duration of the night-time heating period. Therefore, errors

Table 3 Coefficients $a$ and $b$ and $R^{2}$ of the linear relationship between $E_{\Delta T_{t}}$ and $J_{t}$ of Fig. 3 for a night heating period of $3 \mathrm{~h}$

\begin{tabular}{llll}
\hline Day heating period (h) & $a$ & $b$ & $R^{2}$ \\
\hline 0.5 & -1.25 & 0.92 & 0.54 \\
1 & -0.51 & 0.35 & 0.29 \\
1.5 & -0.19 & 0.17 & 0.12 \\
2 & -0.07 & 0.086 & 0.058 \\
\hline
\end{tabular}

during night measurements are one of the reasons for such a bias.

\subsection{Accounting for maximum $\Delta T_{0}$ at night}

When using the longest heating cycles ( 3 or $5 \mathrm{~h}$ ) during the night, we minimised the error in the $\Delta T_{0}$ estimation. However, we ignored night transpiration if it occurred because we considered only one value per night. An additional error should be considered in such a case because a single value may not be enough to identify the maximum $\Delta T$. An analysis of the night course of $\Delta T$ from the continuously heated sensors showed that the maximum night $\Delta T_{0}$ never occurred at the same time (Fig. 4). Thus, when arbitrarily setting the time of night measurement, an additional error is made in the estimation of $\Delta T_{0}$ resulting from not selecting the actual night maximum. For example, this additional error was estimated by the difference between $\Delta T$ at 3:00 a.m. and the actual night maximum $\Delta T$ detected by the continuously heated sensors, $0.08^{\circ} \mathrm{C}$, on average (standard deviation, $0.08^{\circ} \mathrm{C}$ ), representing an error of $0.031 \mathrm{Ldm}^{-2} \mathrm{~h}^{-1}$ under the maximal sap flux density (0.68 $\mathrm{Ldm}^{-2} \mathrm{~h}^{-1}$ ) and of $0.013 \mathrm{Ldm}^{-2} \mathrm{~h}^{-1}$ under the minimal sap flux density $\left(0.017 \mathrm{Ldm}^{-2} \mathrm{~h}^{-1}\right)$. More than $50 \%$ of the night maximal $\Delta T$ occurred at 6:00 a.m., before dawn (Fig. 4), but selecting these data did not decrease the error in $\Delta T_{0}$. Statistically, the minimum error occurred when $\Delta T_{0}$ was measured between 1:00 a.m. and 3:00 a.m. The decreased accuracy when estimations of $\Delta T_{0}$ estimation $\left(0.023^{\circ} \mathrm{C}\right)$ were made at predawn remained low compared to the optimal estimator at 2 a.m. To determine the best time for the measurement of $\Delta T_{0}$, the statistical analysis of the occurrence of the minimum error should be repeated. 
Table 4 Means and standard deviations of the absolute error, bias (mean error) and mean relative error in sap flux density calculations before correction (Eq. 6)

\begin{tabular}{|c|c|c|c|c|c|c|c|c|c|}
\hline \multirow[t]{2}{*}{$\begin{array}{l}\text { Night heating } \\
\text { period }\end{array}$} & \multirow[t]{2}{*}{$\begin{array}{l}\text { Day heating } \\
\text { period }(\mathrm{h})\end{array}$} & \multicolumn{2}{|c|}{$\begin{array}{l}\text { Mean absolute error } \\
\left(\mathrm{L} \mathrm{dm}-2 \mathrm{~h}^{-1}\right)\end{array}$} & \multicolumn{2}{|c|}{$\begin{array}{l}\text { Standard deviation absolute } \\
\text { error }\left(\mathrm{L} \mathrm{dm}-2 \mathrm{~h}^{-1}\right)\end{array}$} & \multicolumn{2}{|c|}{$\operatorname{Bias}\left(L \mathrm{dm}^{-2} \mathrm{~h}^{-1}\right)$} & \multicolumn{2}{|c|}{ Mean relative error } \\
\hline & & Pair 1 & Pair 2 & Pair 1 & Pair 2 & Pair 1 & Pair 2 & Pair 1 & Pair 2 \\
\hline \multirow[t]{4}{*}{$1 \mathrm{~h}$} & 0.5 & 0.076 & 0.062 & 0.044 & 0.044 & -0.039 & 0.013 & 1.81 & 0.77 \\
\hline & 1 & 0.064 & 0.109 & 0.046 & 0.064 & 0.033 & 0.109 & 0.36 & 0.51 \\
\hline & 1.5 & 0.077 & 0.144 & 0.058 & 0.060 & 0.066 & 0.144 & 0.28 & 0.53 \\
\hline & 2 & 0.085 & 0.163 & 0.052 & 0.054 & 0.085 & 0.163 & 0.25 & 0.49 \\
\hline \multirow[t]{4}{*}{$3 \mathrm{~h}$} & 0.5 & 0.152 & 0.124 & 0.075 & 0.060 & -0.152 & -0.123 & 1.74 & 1.69 \\
\hline & 1 & 0.070 & 0.040 & 0.051 & 0.026 & -0.064 & -0.023 & 1.41 & 1.77 \\
\hline & 1.5 & 0.044 & 0.028 & 0.035 & 0.022 & -0.029 & 0.009 & 1.01 & 0.65 \\
\hline & 2 & 0.028 & 0.029 & 0.024 & 0.024 & 0.025 & -0.010 & 0.21 & 0.30 \\
\hline \multirow[t]{4}{*}{$5 \mathrm{~h}$} & 0.5 & 0.172 & 0.163 & 0.079 & 0.054 & -0.172 & -0.163 & 1.94 & 2.12 \\
\hline & 1 & 0.083 & 0.060 & 0.054 & 0.032 & -0.081 & -0.060 & 1.47 & 1.52 \\
\hline & 1.5 & 0.053 & 0.029 & 0.036 & 0.018 & -0.045 & -0.025 & 1.14 & 1.25 \\
\hline & 2 & 0.029 & 0.014 & 0.025 & 0.011 & -0.023 & -0.008 & 0.57 & 0.66 \\
\hline
\end{tabular}

\subsection{Applicability of the corrections on other trees}

The relationship between sap flux density before correction and the slope of the increase in $\Delta T$ during the heating period is an indicator of the trees' thermal properties. A linear relationship $\left(R^{2}=0.6\right)$ fits for all trees at both sites (Fig. 5). Considering the relationships of every tree separately (Fig. 5), the coefficients are not significantly different $(p$ value $>0.9)$. The kinetics of heat exchanges were therefore similar for all the monitored fir trees, allowing us to apply the coefficients of Eqs. 8 and 9 estimated from a single tree to all of the others.

\section{Discussion}

4.1 Performance of the sap flux density correction method

The proposed method demonstrates that Granier's calibration can be used when the appropriate corrections are made to the $\Delta T$ measurements to account for non-stationary regime. It opens the possibility of extrapolating a thermal equilibrium from short heating cycles. We obtained a relative error in sap flux density lower than $30 \%$ in most cases. This experiment was carried out on trees with low sap flux densities during the measurement period; the maximum sap flux density was
Table 5 Means and standard deviations of the absolute error, bias (mean error) and mean relative error in sap flux density calculations after correction (Eq. 7)

\begin{tabular}{lccccc}
\hline $\begin{array}{l}\text { Night heating } \\
\text { period }\end{array}$ & $\begin{array}{l}\text { Day heating } \\
\text { period (h) }\end{array}$ & $\begin{array}{l}\text { Mean absolute } \\
\text { error }\left(\mathrm{L} \mathrm{dm}^{-2} \mathrm{~h}^{-1}\right)\end{array}$ & $\begin{array}{l}\text { Absolute error } \\
\text { standard deviation } \\
\left(\mathrm{L} \mathrm{dm}^{-2} \mathrm{~h}^{-1}\right)\end{array}$ & $\begin{array}{l}\text { Bias } \\
\left(\mathrm{L} \mathrm{dm}^{-2} \mathrm{~h}^{-1}\right)\end{array}$ & $\begin{array}{l}\text { Mean relative } \\
\text { error }\end{array}$ \\
\hline $1 \mathrm{~h}$ & 0.5 & 0.031 & 0.031 & 0.026 & 0.51 \\
& 1 & 0.040 & 0.033 & 0.035 & 0.24 \\
& 1.5 & 0.042 & 0.034 & 0.041 & 0.19 \\
$3 \mathrm{~h}$ & 2 & 0.030 & 0.027 & 0.024 & 0.27 \\
& 0.5 & 0.032 & 0.028 & 0.017 & 0.21 \\
& 1 & 0.026 & 0.024 & 0.018 & 0.26 \\
$5 \mathrm{~h}$ & 1.5 & 0.026 & 0.024 & 0.021 & 0.23 \\
& 2 & 0.025 & 0.021 & 0.020 & 0.21 \\
& 0.5 & 0.030 & 0.029 & -0.010 & 0.55 \\
& 1 & 0.021 & 0.018 & 0.011 & 0.35 \\
& 1.5 & 0.021 & 0.018 & 0.016 & 0.20 \\
\hline
\end{tabular}




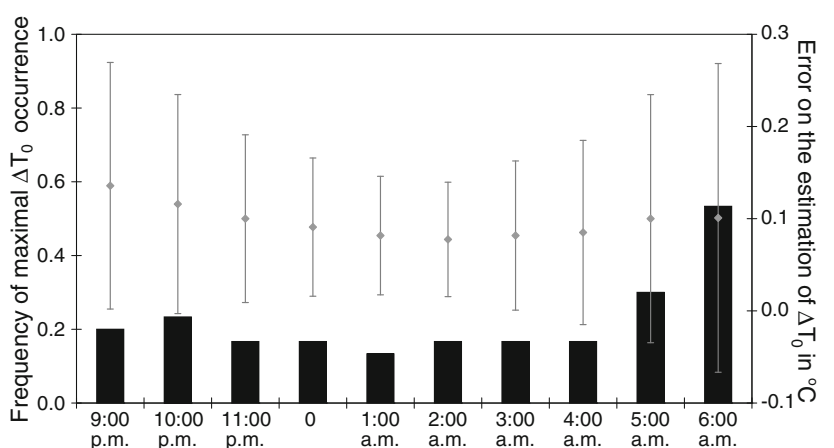

Fig. 4 Frequency of the maximal $\Delta T_{0}$ occurring for each night period, measured from continuously heated sensors (black bars). Maximums can occur at different times of night. Error is calculated by comparison to maximal $\Delta T_{0}$ and is represented by grey points

$0.68 \mathrm{Ldm}^{-2} \mathrm{~h}^{-1}$, whereas it is typically between 1 and $2 \mathrm{~L}$ $\mathrm{dm}^{-2} \mathrm{~h}^{-1}$ for coniferous trees (Lu et al. 1996; Köstner et al. 1998; Čermák et al. 2004; Delzon and Loustau 2005; Nadezhdina et al. 2007). The error before correction would have been lower with higher fluxes. Indeed, in our case, the length of time necessary to reach a stationary regime was long.

Furthermore, applying a long heating period during the night, even if it reduces the remaining error, limits data collection to a single value per night. As a result, it is not possible to detect night transpiration, leading to an increase in the risk of error when night transpiration occurs at the time of measurement. In the end, the duration of heating should be chosen based on a trade-off between time resolution, precision after correction and energy saving. One recommendation is to use a 5 -h heating cycle during the night, with $\Delta T_{0}$ measured at predawn, and a $1-\mathrm{h}$ heating and 1-h cooling cycle during the day to minimise bias and absolute error, and to have a rather good time resolution during the day. Additionally, to guarantee a valid correction, attention should be paid to the choice of sensors that form pairs on trees with the same sap flux dynamics. This aspect of the method can compromise its precision. When

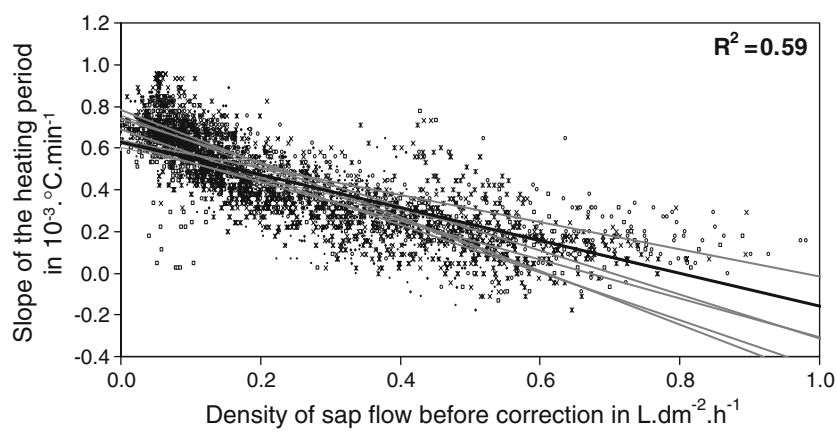

Fig. 5 Relationship between the slope of the heating period (difference of measured $\Delta T$ between $15 \mathrm{~min}$ and $1 \mathrm{~h}$ of heating) and sap flux density (calculated without correction) for each tree. The black line corresponds to the linear relationship for all trees together the temporal pattern of sensors does really not match, the correction is far less accurate (results not shown).

The applicability of the relationship established has been validated for the silver fir trees of Mont Ventoux, but it is not certain whether it is suitable for fir trees with higher growth rates or different trunk diameters or for other tree species. The strength of the proposed method is that it can be implemented on existing experimental setups by simply changing the configuration of the acquisition protocol to have one or several pairs of sensors. It is therefore easy to repeat the method, collect references on the correction coefficients $a, b$ and $c$ and analyse their variations in relation to tree characteristics. An experiment in the laboratory with a controlled $J_{t}$ would be interesting to test the hypothesis of reaching the stationary regime at $t_{m}$ and adding accuracy to the calibration.

\subsection{Comparison with the D\&R calibration}

In addition to the proposed correction method, we computed sap flow densities using the D\&R calibration on the cyclic sensor and using Granier's calibration on the continuous sensor of pair 2 to obtain a reference sap flux density. The D\&R calibration was applied using $\Delta T$ measurements after $0.5 \mathrm{~h}$ of heating and cooling to reproduce D\&R heating protocols. To be as close as possible to the D\&R heating protocol, we applied the proposed correction method with measurements from a cycle of $0.5 \mathrm{~h}$ of heating during the day and $1 \mathrm{~h}$ at night. The results are displayed in Fig. 6. There is a clear overestimation when the calculation is done with D\&R calibration and a poor relationship $\left(R^{2}=0.52\right)$ with the reference measurements. This overestimation was already observed by Isarangkool $\mathrm{Na}$ Ayutthaya et al. (2010) for low sap flow rates, but the difference was much smaller. The sap

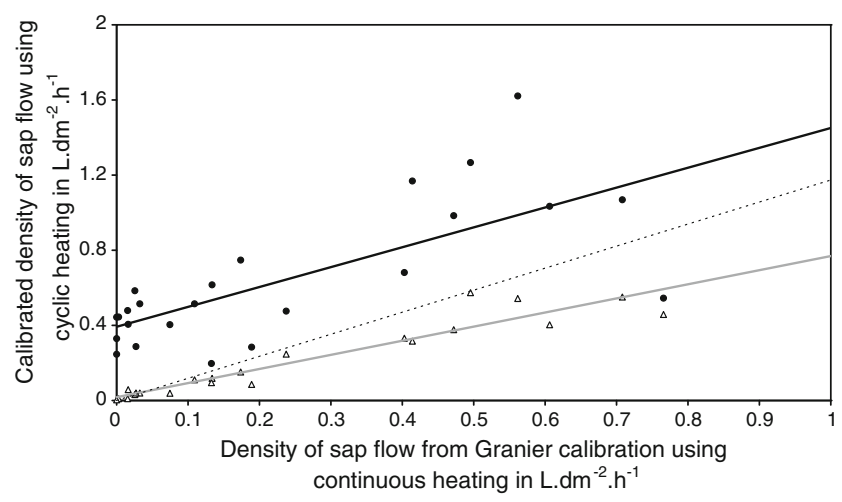

Fig. 6 Relationships between the sap flux density using a continuous heating and Granier's calibration and (1) the sap flux density using a cyclic heating calculated based on the proposed method (triangles and grey continuous lines) and (2) the sap flux density calculated based on D\&R calibration (black points and black linear regression). Cyclic measurements were performed using a heating period of $0.5 \mathrm{~h}$ during daylight and $0.5 \mathrm{~h}$ during the night to implement D\&R and 0.5 and $1 \mathrm{~h}$ for the proposed method 
flux densities obtained with the proposed method were correlated closely to the reference $\left(R^{2}=0.90\right)$, showing the efficiency of the calibration procedure. The purpose here is not to compare a calibrated (the proposed method) to an uncalibrated (D\&R) method, but to highlight that the wood temperature dynamic under cyclic heating can depend on the sap flux density rates and on the tree species. Therefore, care should be taken in applying the $\mathrm{D} \& \mathrm{R}$ calibration in different contexts.

Do and Rocheteau (2002b) also highlighted the importance of the cyclic method to account for the natural temperature gradients. We took advantage of two severalday periods without heating to estimate these gradients: a hot period in July and a cooler one in May. There were significant gradients, and they presented daily cycles. The cycles were variable from one day to another and between sensors. The mean natural gradients were $0.16^{\circ} \mathrm{C}$ in July and $0.125^{\circ} \mathrm{C}$ in May. It is possible to measure them at the end of the cooling period when using cyclic heating. The question is whether equilibrium is attained and if the $\Delta T$ measured at the end of the cooling phase reflects the natural temperature gradients. In July, the thermal equilibrium at the end of the cooling period was attained faster as fluxes were higher, and the mean $\Delta T$ measured (after $1 \mathrm{~h}$ of cooling) at the end of the cooling phase was $0.20^{\circ} \mathrm{C}$. In this case, the natural gradients represented $78 \%$ of the $\Delta T$ measured. In May, the $\Delta T$ measured at the end of the cooling phase was higher $\left(0.25^{\circ} \mathrm{C}\right)$, as the equilibrium is not reached, and the proportion of the natural gradient in $\Delta T$ was lower $(50 \%)$. Accounting for these natural gradients can be accomplished when calculating the corrected sap flux density by subtracting, as proposed by Do and Rocheteau (2002a), values of $\Delta T_{\text {off }}$ to each $\Delta T$ of Granier's equation. When applying this to the sap flux density calculation (with correction for a cycle of 5-h heating and 3-h cooling during the night, and 2-h heating and 2$\mathrm{h}$ cooling during daylight), the mean difference with the initial corrected calculation was $0.037 \mathrm{Ldm}^{-2} \mathrm{~h}^{-1}$ (standard deviation, $0.047 \mathrm{Ldm}^{-2} \mathrm{~h}^{-1}$ ), with a positive or a negative bias depending on the sensor. In our experiment, the natural temperature gradients had a moderate influence on the sap flux density calculation. However, that may have been interesting to take them into account. With this perspective, the proposed cyclic method offers advantageous possibilities in comparison to the classical Granier approach.

\section{Conclusion}

Cyclic heating is of great interest for saving energy, an issue that can be a limiting factor in some experiments. If short heating cycles are applied, the stationary regime is not always reached, leading to errors in sap flux calculations. The correction method is easy to implement and the calibration period must cover the encountered range of sap flux density. The only constraint is to implement two heating protocols concurrently during the calibration period because the calibration protocol is based on a pair of sensors. Once calibrated, the correction method can be applied to all sensors under similar heating cycles in trees of the same species but located at different places in a forest, and short heating cycles can be used. The proposed correction method is flexible in terms of heating cycle characteristics but should be calibrated for each cycle characteristics. As the correction calibration can be repeated easily, its validity can be established by considering other tree species.

Acknowledgments We thank G. Sappe and D. Gounelle for their help in constructing the sensors and B. Bes for his help in the field. The paper was significantly improved thanks to the remarks and suggestions from Dr. P. Lu and from an anonymous reviewer.

Funding The research was funded by the Agence Nationale de Recherche (project ANR-06-VULN-004) and by the Office National des Forêts $(\mathrm{ONF})$ by the bias of a $\mathrm{PhD}$ grant.

\section{References}

Andrade JL, Meinzer FC, Golstein G, Holbrook NM, Cavelier J, Jackson P, Sivera K (1998) Regulation of water flux through trunks, branches, and leaves in trees of a lowland tropical forest. Oecologia 115:463-471

Braun P, Schmid J (1999) Sap flow measurements in grapevines (Vitis vinifera L.) 2. Granier measurements. Plant Soil 215:47-55

Čermák J, Kučera J, Nadezhdina N (2004) Sap flow measurements with some thermodynamic methods, flow integration within trees and scaling up from sample trees to entire forest stands. Trees 18:529-546

Delzon S, Loustau D (2005) Age-related decline in stand water use: sap flow and transpiration in a pine forest chronosequence. Agric For Meteorol 129:105-119

Do F, Rocheteau A (2002a) Influence of natural temperature gradients on measurements of xylem sap flow with thermal dissipation probes. 1 . Field observations and possible remedies. Tree Physiol 22:641-648

Do F, Rocheteau A (2002b) Influence of natural temperature gradients on measurements of xylem sap flow with thermal dissipation probes. 2. Advantages and calibration of non continuous heating system. Tree Physiol 22:649-653

Granier A (1985) A new method of sap flow measurement in tree stems. Ann For Sci 42:193-200

Granier A (1987) Sap flow measurements in Douglas fir tree trunks by means of a new thermal method. Ann For Sci 44(1):1-14

Isarangkool Na Ayutthaya S, Do FC, Pannengpetch K, Junjittakarn J, Maeght J-L, Rocheteau A, Cochard H (2010) Transient thermal dissipation method of xylem sap flow measurement: multispecies calibration and field evaluation. Tree Physiol 30:139-148

Köstner B, Granier A, Cermak J (1998) Sapflow measurements in forest stands: methods and uncertainties. Ann For Sci 55:1327

Lu P, Biron P, Granier A, Cochard H (1996) Water relations of adult Norway spruce (Picea abies (L.) Karst) under soil drought in the Vosges mountains: whole-tree hydraulic conductance, xylem embolism and water loss regulation. Ann For Sci 53(1):113-121 
Lu P, Urban L, Zhao P (2004) Granier's thermal dissipation probe (TDP) method for measuring sap flow in trees: theory and practice. Acta Bot Sin 46(6):631-646
Nadezhdina N, Cermak J, Meiresonne L, Ceulemans R (2007) Transpiration of Scots pine in Flanders growing on soil with irregular substratum. For Ecol Manag 243:1-9 